\title{
Elevated OPN, IP-10, and Neutrophilia in Loop-Mediated Isothermal Amplification Confirmed Tuberculosis Patients
}

\author{
Beata Shiratori, ${ }^{1,2,3}$ Susan Leano, ${ }^{4}$ Chie Nakajima, ${ }^{5}$ Haorile Chagan-Yasutan, ${ }^{1,2}$ \\ Toshiro Niki, ${ }^{6}$ Yugo Ashino, ${ }^{1,2}$ Yasuhiko Suzuki, ${ }^{5}$ Elisabeth Telan, ${ }^{4}$ and Toshio Hattori ${ }^{1,2,7}$ \\ ${ }^{1}$ Division of Disaster-Related Infectious Diseases, International Research Institute of Disaster Science, Tohoku University, \\ 1-1 Seiryo-machi, Aoba-ku, Sendai, Miyagi 980-8574, Japan \\ ${ }^{2}$ Division of Emerging Infectious Diseases, Graduate School of Medicine, Tohoku University, 1-1 Seiryo-machi, Aoba-ku, \\ Sendai, Miyagi 980-8574, Japan \\ ${ }^{3}$ Japan International Corporation of Welfare Services, 2-3-20 Toranomon YHK Building 4F, Toranomon, Minato-ku, \\ Tokyo 105-0001, Japan \\ ${ }^{4}$ STD AIDS Cooperative Central Laboratory, San Lazaro Hospital, Quiricada Street, 1003 Manila, Philippines \\ ${ }^{5}$ Division of Global Epidemiology, Research Center for Zoonosis Control, Hokkaido University, North 20, \\ West 10 Kita-ku, Sapporo 001-0020, Japan \\ ${ }^{6}$ Research Division, GalPharma Company, Ltd., NEXT-Kagawa 204, 2217-44 Hayashi-cho, Takamatsu-shi, Kagawa 760-0301, Japan \\ ${ }^{7}$ Division of Disaster-Related Infectious Diseases, International Research Institute of Disaster Science, Tohoku University, \\ 2-1 Seiryo-machi, Aoba-ku, Sendai 980-8574, Japan
}

Correspondence should be addressed to Toshio Hattori; toshatto@med.tohoku.ac.jp

Received 24 June 2014; Revised 21 August 2014; Accepted 8 September 2014; Published 15 October 2014

Academic Editor: Sandra Helena Penha Oliveira

Copyright (C) 2014 Beata Shiratori et al. This is an open access article distributed under the Creative Commons Attribution License, which permits unrestricted use, distribution, and reproduction in any medium, provided the original work is properly cited.

\begin{abstract}
Tuberculosis (TB) is the second most common cause of death from infectious diseases and results in high socioeconomic losses to many countries. Proper diagnosis is the first step in TB eradication. To develop a rapid, simple, and accurate diagnostic TB test and to characterize the prevalence of Mycobacterium tuberculosis (MTB) genotypes and immune profiles of TB patients, a total of $37 \mathrm{~TB}$ patients and 30 healthy control (HC) from Metro Manila were enrolled. Loop-mediated isothermal amplification (LAMP) reliably detected MTB infection. Manila genotype was identified by spoligotyping method in all TB patients. Osteopontin (OPN), interferon- $\gamma$-induced protein $10 \mathrm{kDa}$ (IP-10), and neutrophil counts were found to reflect the acute stage of MTB infection. The sensitivity and specificity were $94.6 \%$ and $93.3 \%$, respectively, for both OPN and IP-10, and they were $83.8 \%$ and $78.6 \%$, respectively, for neutrophils. The combination of OPN, IP-10, neutrophil count, IL-6, IL-8, TNF- $\alpha$, MCP-1, platelets, galectin-9, and leukocyte count correctly identifies all the HC and $96.3 \%$ of TB patients. LAMP method may serve as a rapid, supportive method in addition to time-consuming culture methods. OPN, IP-10, and neutrophil counts are useful in detecting MTB infection and may have utility in monitoring the course of the disease.
\end{abstract}

\section{Introduction}

Mycobacterium tuberculosis (MTB) is the causative agent of tuberculosis (TB), the sixth leading cause of death and illness in the Philippines. Despite global trends towards decreasing incidence, prevalence, and mortality associated with MTB infection, approximately 230,000 cases were found in the Philippines alone in 2012 [1]. The elderly, urban poor, smokers, and individuals with compromised immune systems, such as those living with HIV, malnutrition, and diabetes, belong to the high-risk group [2]. Beyond the health burdens associated with MTB, serious socioeconomic losses are another consequence of this disease.

Developing a rapid, simple, and accurate test for $\mathrm{TB}$ diagnosis is a main focus of many investigators. In lowresource countries, TB is most often diagnosed based on clinical symptoms, radiographic features, and microscopic observation of acid fast bacillus (AFB). Bacterial culture methods require long culturing time to obtain acceptable sensitivity, which delays early initiation of anti-TB treatment. 
In recent years, nucleic acid amplification (NAA) tests have shown potential as the optimal $\mathrm{TB}$ diagnostic approach for MTB diagnosis. Loop-mediated isothermal amplification (LAMP) is an NAA method that enables the detection of trace amounts of bacterial DNA under isothermal conditions within one hour [3].

Osteopontin (OPN) is a multifunctional phosphorylated glycoprotein that is synthesized by a variety of immune and nonimmune cells $[4,5]$. Increased levels of OPN have been observed during MTB infection and other infectious pathogens $[4,6,7]$, polarizing the immune response towards a $T_{h} 1$ response through the enhancement of IL-12 and IFN- $\gamma$ secretion $[4,8]$.

Galectin-9 (gal-9) is a $\beta$-galactoside-binding matricellular protein that induces cell activation, chemoattraction, and cell death. Gal-9, binding to its receptor, T-cell immunoglobulin and mucin domain-containing molecule-3 (Tim-3), stimulates bactericidal activity in mouse TB models $[9,10]$. Gal-9 and Tim-3 expression in CD4+ and CD8+ T-cells may be elevated during TB infection in humans, compared to healthy individuals [11]; however, their concentrations in plasma have not been reported until now.

Interferon- $\gamma$ induced protein $10 \mathrm{kDa}$ (IP-10) is one of the most well studied biomarkers in TB infection and is a promising alternative marker for replacing current interferon- $\gamma$ releasing assay-based methods [12]. IP-10 is involved in multiple biological functions, inducing chemotaxis, apoptosis, inhibition of cell growth, and recruiting activated T-cells, macrophages, and NK cells to sides of infection [12].

Relatively little attention has been paid to the role of neutrophils during MTB infection, compared to macrophages and other host immune response components. Neutrophils elicit strong phagocytic activity [13] and neutrophil-driven, interferon (IFN)-inducible transcript signatures in whole human blood, which were recently shown to be associated with disease severity, suggesting a direct contribution of neutrophils to pathogenesis [14]. Other studies have demonstrated that neutrophils contribute to early defense responses against MTB [15, 16], but in later stages of the disease, an opposite tendency is observed $[17,18]$.

In the present study, we evaluated the reliability of LAMP for detecting MTB infection and used spoligotyping to identify the most prevalent MTB genotype in Metro Manila. We also analyzed a broad spectrum of biomarkers, which reflect both cellular and humoral immune response to MTB infection. Our results confirmed the utility of the LAMP and spoligotyping methods for TB diagnostics and genotyping and showed that OPN, IP-10, and neutrophil counts reflect the acute stage of disease and are promising biomarkers to monitor the course of the disease.

\section{Materials and Methods}

2.1. Participants. The study population consisted of 37 HIVnegative patients randomly selected from the out-patient department of San Lazaro Hospital (SLH, Manila, Philippines) who had positive AFB staining, clinical symptoms, and chest radiographs characteristic of pulmonary TB and had no prior history of TB treatment. The controls were healthy volunteers lacking signs of TB; however, their histories of latent tuberculosis infection (LTBI) or other pulmonary diseases are unknown. The study was approved by the ethics committee of SLH and the Tohoku University Hospital, and written informed consent was obtained from each participant.

2.2. Sample Collection. Blood was collected in $5 \mathrm{~mL}$ EDTA tubes and plasma was separated by centrifugation and stored at $-80^{\circ} \mathrm{C}$ until analyzed. Sputa were decontaminated by conventional procedures using NALC-NaOH. After centrifugation, supernatants were discarded and DNA was released from cell pellets by heating at $95^{\circ} \mathrm{C}$ for $5 \mathrm{~min}$, which was repeated three times.

2.3. Clinical Data and Biomarker Measurement. Complete blood counts $(\mathrm{CBC})$, including differential counts of white blood cells and plasma levels of $\operatorname{IgG}$ and $\operatorname{IgA}$, were performed for samples from each individual. Plasma levels of 29 cytokines and chemokines were measured using the Milliplex MAP (Millipore, Germany). The Bio-Plex Manager Software (version 6.0) was used for bead acquisition and analysis. OPN protein levels were measured using the Human Osteopontin Quantikine ELISA Kit (R\&D Systems, USA) and gal-9 levels by an ELISA kit (GalPharma, Japan). Antitubercular glycolipid (TBGL) IgG and IgA antibody titers were obtained using the Determiner TBGL Antibody ELISA Kit (Kyowa Medex, Japan).

2.4. LAMP Method. LAMP reactions were performed in reaction mixture $(25 \mu \mathrm{L})$ containing 6 primers [3], $1.4 \mathrm{mM}$ deoxynucleoside triphosphates, $0.8 \mathrm{M}$ betaine, $20 \mathrm{mM}$ Tris/HCl (pH 8.8), $10 \mathrm{mM} \mathrm{KCl}, 10 \mathrm{mM}\left(\mathrm{NH}_{4}\right)_{2} \mathrm{SO}_{4}, 8 \mathrm{mM}$ $\mathrm{MgSO}_{4}, 8 \mathrm{U}$ Bst DNA polymerase (New England Biolabs), and $5 \mu \mathrm{L}$ of bacterial DNA. The mixture was incubated at $64^{\circ} \mathrm{C}$ for $60 \mathrm{~min}$ in a Loopamp real-time turbidimeter (LA-200, Teramecs). Reactions were considered positive when turbidity was greater than $0.1(650 \mathrm{~nm})$ within $60 \mathrm{~min}$.

2.5. Spoligotyping Method. Spoligotypes of clinical MTB isolates were determined as described previously [19]. Briefly, the DR region was amplified with a primer pair, and the PCR products were hybridized to a set of 43 spacer-specific oligonucleotide probes, which were covalently bound to membranes. The spoligo-international type was determined by comparing spoligotypes against the international spoligotyping database [20].

2.6. Statistical Analysis. The Mann-Whitney $U$-test was used to compare the differences between the two groups, and the Spearman's rank correlation was used to analyze relationships between biomarkers and other parameters. Statistical analyses were performed using the GraphPad Prism program (version 6, GraphPad Software, USA) and the SPSS program (version 22, SPSS Inc., USA). Discriminant function analyses and Receiver operator characteristic (ROC) curve analyses were used to evaluate the predictive capacities of biomarkers. Area under the curve (AUC) values were calculated, and cut-off values were estimated based on the best proportion 
TABLE 1: Characteristics of HC and TB individuals.

\begin{tabular}{|c|c|c|c|}
\hline & $\mathrm{HC}(n=30)$ & TB $(n=37)$ & $P$ value \\
\hline \multicolumn{4}{|l|}{ Antropometric data } \\
\hline Age year; median (range) & $28.5(22-59)$ & $40(18-60)$ & ns \\
\hline Gender: male; $n(\%)$ & $9(30)$ & $23(62.2)$ & $* *$ \\
\hline BMI & na & $20.42(14-44.5)$ & \\
\hline Employed (\%) & 100 & 48.4 & \\
\hline Had contact with TB (\%) & 0 & 48.6 & \\
\hline \multicolumn{4}{|l|}{ Clinical symptoms } \\
\hline Cough $(\%)$ & na & 94.4 & \\
\hline Fever $(\%)$ & na & 77.8 & \\
\hline Sweat (\%) & na & 66.7 & \\
\hline Loss of appetite (\%) & na & 25 & \\
\hline \multicolumn{4}{|l|}{ Radiographic features } \\
\hline \multicolumn{4}{|l|}{ Site of pathological finding } \\
\hline One lung (\%) & na & 31.2 & \\
\hline Both lungs (\%) & na & 68.8 & \\
\hline Cavity (\%) & na & 46.9 & \\
\hline Effusion (\%) & na & 6.3 & \\
\hline \multicolumn{4}{|c|}{ Laboratory findings: median (range) } \\
\hline $\operatorname{RBC}\left(10^{6} / \mu \mathrm{L}\right)$ & $4.8(4.3-6.5)$ & $4.6(3-6.2)$ & $*$ \\
\hline Hemoglobin (g/dL) & $13.8(12.2-17.2)$ & $12.5(8.3-16)$ & $* * * *$ \\
\hline Hematocrit (\%) & $42.2(39.1-51.8)$ & $37.8(25.6-49.6)$ & $* * * *$ \\
\hline $\mathrm{WBC}\left(10^{3} / \mu \mathrm{L}\right)$ & $6.8(4.4-11.8)$ & $9.1(4.4-19.6)$ & $* * * *$ \\
\hline Neutrophil (\%) & $52.5(37-67)$ & $70(49-85)$ & $* * * *$ \\
\hline Lymphocyte (\%) & $38.5(24-68)$ & $19(6-33)$ & $* * * *$ \\
\hline Monocyte (\%) & $5(2-11)$ & $8(3-13)$ & $* * * *$ \\
\hline Eosinophil (\%) & $3.5(1-8)$ & $3(1-10)$ & ns \\
\hline Platelet $\left(10^{3} / \mu \mathrm{L}\right)$ & $305.5(178-560)$ & $467(143-784)$ & $* * * *$ \\
\hline Plasma IgG (mg/dL) & $1134(659-3266)$ & $1618(934-2456)$ & $* *$ \\
\hline Plasma IgA (mg/dL) & $329(115-973)$ & $512(162-1126)$ & $* *$ \\
\hline
\end{tabular}

${ }^{*} P<0.05,{ }^{* *} P<0.01,{ }^{* * *} P<0.001,{ }^{* * * *} P<0.0001$.

TB group versus $\mathrm{HC}$ group by Mann-Whitney $U$-test.

ns: not significant, na: not applicable.

between sensitivity, specificity, and likelihood ratio. $P$ values $<0.05$ were considered statistically significant.

\section{Results}

3.1. Characteristics of Study Participants. A total of $37 \mathrm{~TB}$ patients and 30 healthy controls (HC) were enrolled in this study. Basic anthropometric and clinical characteristics of study participants are shown in Table 1 . Chest lung Xrays showed bilateral pathology in $68.8 \%$ of cases, and only one lung was affected in $31.2 \%$ of cases. Lung cavities and effusion were found in $47 \%$ and $6.3 \%$ of TB patients, respectively. Laboratory findings revealed lower red blood counts, hemoglobin levels, hematocrits, and relative lymphocyte counts in the TB group compared to the HC group. Absolute numbers of white blood cells (WBC) and relative counts of neutrophils, monocytes, platelets, and plasma IgG and IgA were higher in TB patients (Table 1). The absolute and relative values of each type of WBC were strongly correlated (data not shown).

3.2. LAMP and Genotyping. We confirmed MTB infection in all 37 patients' samples by the LAMP method. All DNA samples were subjected to spoligotyping to identify the most prevalent genotype. We found that all patients were infected with a Manila type MTB.

\subsection{Comparison of Plasma Biomarker Concentrations between} $T B$ and HC Individuals. Using a multiplex immunoassay, we assessed the concentrations of 29 soluble plasma biomarkers in $\mathrm{HC}$, as well as TB patients before antituberculous therapy was initiated. Biomarker levels and their comparisons between groups are shown in Table 2. Significantly elevated concentrations of IL- $1 \alpha$, TNF- $\alpha$, IL-6, eotaxin, IL-8, IP-10, and MCP-1 were observed with the TB group. IL- $1 \beta$, IL2, IL-3, IL-15, IL-17A, IL-4, IL-5, IL-13, MIP-1 $\alpha$, TNF- $\beta$, and IL-10 levels were not considered for statistical analyses 
TABLE 2: Comparison of cellular and humoral immunity biomarkers between HC and TB individuals.

\begin{tabular}{|c|c|c|c|}
\hline $\begin{array}{l}\text { Biomarker } \\
(\mathrm{pg} / \mathrm{mL})\end{array}$ & \multicolumn{2}{|c|}{ Median (range) } & $P$ \\
\hline \multicolumn{4}{|l|}{ General activation } \\
\hline IL-1 $\alpha$ & $0(0-51.7)$ & $6.8(0-40.7)$ & $* *$ \\
\hline IL-1RA & $39.7(0-374.9)$ & $34.7(0-230.9)$ & ns \\
\hline TNF- $\alpha$ & $4.2(0-15.2)$ & $9.6(0-60.5)$ & $* * * *$ \\
\hline IFN- $\alpha$ & $40.6(0-128.5)$ & $23.6(3.9-157.9)$ & ns \\
\hline IL-6 & $0(0-23.4)$ & $6.6(0-41.4)$ & $* * * *$ \\
\hline \multicolumn{4}{|l|}{ Th1 related } \\
\hline IFN- $\gamma$ & $9.3(0-35.2)$ & $12.7(0-60.8)$ & ns \\
\hline IL-12p40 & $31.4(0-140.6)$ & $14(0-249)$ & ns \\
\hline IL-12p70 & $13.5(0-73.9)$ & $6.7(0-58.2)$ & ns \\
\hline \multicolumn{4}{|l|}{ Bone marrow derived } \\
\hline IL-7 & $11(0-34.9)$ & $9.6(0-25.4)$ & ns \\
\hline GM-CSF & $7.6(0.8-23.0)$ & $5.1(0-49.2)$ & ns \\
\hline G-CSF & $144.1(19.3-458.0)$ & $129.4(0-310.4)$ & ns \\
\hline \multicolumn{4}{|l|}{ Stromal, angiogenic } \\
\hline VEGF & $128(0-344.1)$ & $178.4(0-1287.3)$ & ns \\
\hline EGF & $109.8(42.3-488.2)$ & $109.8(0-459.5)$ & ns \\
\hline \multicolumn{4}{|l|}{ Chemokine } \\
\hline IL-8 & $2.3(0-10)$ & $8(0-61.1)$ & $* * * *$ \\
\hline IP-10 & $242.6(97.8-453.3)$ & $1290(0-9235)$ & $* * * *$ \\
\hline MCP-1 & $87.1(48.7-154.5)$ & $128.5(41.6-280.5)$ & $* * * *$ \\
\hline MIP-1 $\beta$ & $31.9(0-51.9)$ & $33.3(0-58.8)$ & ns \\
\hline Eotaxin & $38.5(18.1-112.6)$ & $48(20.7-133.8)$ & * \\
\hline \multicolumn{4}{|l|}{ Matricellular protein } \\
\hline OPN (ng/mL) & $69(40-118.9)$ & $159(28.9-256)$ & $* * * *$ \\
\hline Gal-9 & $195.8(108-507.6)$ & $377(0-2181)$ & $* * * *$ \\
\hline \multicolumn{4}{|l|}{ Antibody } \\
\hline Anti-TBGL IgG (U/mL) & $2.3(0.5-37.3)$ & $9.3(0-64.2)$ & $* *$ \\
\hline Anti-TBGL IgA (U/mL) & $1(0.2-76)$ & $2.6(0-57.6)$ & * \\
\hline
\end{tabular}

${ }^{*} P<0.05,{ }^{* *} P<0.01,{ }^{* * *} P<0.001,{ }^{* * * *} P<0.0001$, ns: not significant.

TB group versus HC group by Mann-Whitney $U$-test.

because their median was below minimum detectable levels in both groups. We measured also the plasma levels of two matricellular proteins, OPN and gal-9, and found statistically higher levels of both proteins in TB patients compared to $\mathrm{HC}$ individuals. Higher titers of TBGL IgG and IgA were found in TB patients. In $\mathrm{HC}$, antitubercular antibodies were correlated with age, anti-TBGL IgG $(r=0.66, P<0.00001)$, and IgA and $(r=0.327, P<0.05)$; however, this relationship was not observed in the TB group (data not shown). Anti-TBGL IgG correlated with anti-TBGL IgA $(r=0.333, P<0.05)$ in the TB group but not in the HC group (data not shown). Anti-TBGL antibody elevation was not related to a general elevation of antibodies in the plasma (data not shown).

3.4. Correlation of OPN, IP-10, Neutrophil Count, and Gal-9 in HC and TB Groups. We performed correlation analyses of the three most predictive TB markers with other measured parameters. IP-10 expression levels positively correlated with loss of appetite, IL-1 $\alpha$, IL-6, IL-8, OPN, and gal-9 expression and negatively correlated with anti-TBGL IgG antibody titer (Table 3 ). In addition, OPN and IL-8 expression levels were correlated (Table 3). TB patients' neutrophilia was associated with increased WBC counts, IFN $-\gamma$, and decreased hemoglobin levels, hematocrits, plasma IgG titers, and lymphocytopenia (Table 3). Finally, we studied the relationship of gal-9 to biomarkers and other laboratory parameters. High gal-9 levels correlated with higher TNF- $\alpha$, IL-6, IL-5, IL-3, EGF, IL-8, and IP-10 expression and lower levels of GM-CSF (Table 3).

3.5. Discrimination Potential of Biomarkers. Biomarkers that differentiated $\mathrm{HC}$ and TB were analyzed by ROC analysis and cut-off values were determined based on the best proportion between sensitivity, specificity, and likelihood ratio (Table 4). The highest discriminatory property had IP-10 and OPN, followed by neutrophils, platelets, TNF- $\alpha$, MCP-1, leukocyte counts, gal-9, and IL-8. Next, we performed analysis, which is a multivariate discrimination method to characterize the 
TABLE 3: Correlation analysis of IP-10, neutrophil, OPN, and gal-9 with other biomarkers in TB patients.

\begin{tabular}{|c|c|c|c|c|c|c|c|c|}
\hline \multirow{2}{*}{$\mathrm{CBC} /$ clinical parameter/biomarker } & \multicolumn{2}{|c|}{ IP-10 } & \multicolumn{2}{|c|}{ Neutrophil } & \multicolumn{2}{|c|}{$\mathrm{OPN}$} & \multicolumn{2}{|c|}{ Gal-9 } \\
\hline & $r$ & $P$ & $r$ & $P$ & $r$ & $P$ & $r$ & $P$ \\
\hline \multicolumn{9}{|l|}{$\mathrm{CBC}$} \\
\hline Hemoglobin & & & -0.375 & * & & & & \\
\hline Hematocrit & & & -0.415 & * & & & & \\
\hline WBC & & & 0.684 & $* * * *$ & & & & \\
\hline Lymphocyte & & & -0.931 & $* * * *$ & & & & \\
\hline Plasma IgG & & & -0.455 & * & & & & \\
\hline \multicolumn{9}{|l|}{ Clinical parameter } \\
\hline Loss of appetite & 0.466 & $* *$ & & & & & & \\
\hline \multicolumn{9}{|l|}{ General activation } \\
\hline IL- $1 \alpha$ & 0.687 & $* * * *$ & & & & & & \\
\hline TNF- $\alpha$ & & & & & & & 0.39 & $* *$ \\
\hline IL-6 & 0.367 & * & & & & & 0.309 & $*$ \\
\hline \multicolumn{9}{|l|}{ Th1 related } \\
\hline IFN- $\gamma$ & & & 0.364 & $*$ & & & & \\
\hline \multicolumn{9}{|l|}{ Bone marrow derived } \\
\hline GM-CSF & & & & & & & -0.335 & $*$ \\
\hline \multicolumn{9}{|l|}{ Chemokine } \\
\hline IL-8 & 0.4635 & $* *$ & & & 0.44 & $* *$ & 0.4635 & $* *$ \\
\hline IP-10 & & & & & 0.422 & $* *$ & 0.295 & $*$ \\
\hline \multicolumn{9}{|l|}{ Matricellular protein } \\
\hline OPN & 0.422 & $* *$ & & & & & & \\
\hline Gal-9 & 0.393 & * & & & & & & \\
\hline \multicolumn{9}{|l|}{ Antibody } \\
\hline Anti-TBGL IgG & -0.423 & $* *$ & & & & & & \\
\hline
\end{tabular}

TABLE 4: Statistic data of ROC analysis of studied biomarkers.

\begin{tabular}{|c|c|c|c|c|c|c|}
\hline Biomarker & AUC & SE & Cut-off & $\begin{array}{c}\text { Sensitivity } \\
\%\end{array}$ & $\begin{array}{c}\text { Specificity } \\
\%\end{array}$ & $\begin{array}{l}\text { Likelihood } \\
\text { ratio }\end{array}$ \\
\hline IP-10 & 0.987 & 0.009 & $>342$ & 94.6 & 93.3 & 14.1 \\
\hline OPN & 0.966 & 0.027 & $>94$ & 94.6 & 93.3 & 14.1 \\
\hline Neutrophil & 0.905 & 0.036 & $>57.5$ & 83.8 & 78.6 & 3.9 \\
\hline Platelet & 0.799 & 0.055 & $>339$ & 75.7 & 80 & 3.8 \\
\hline TNF- $\alpha$ & 0.796 & 0.057 & $>5.99$ & 81.1 & 70 & 2.7 \\
\hline MCP-1 & 0.786 & 0.056 & $>92$ & 75.7 & 63.3 & 2.1 \\
\hline WBC & 0.784 & 0.057 & $>7.5$ & 81.1 & 70 & 2.7 \\
\hline Gal-9 & 0.766 & 0.063 & $>258$ & 75.7 & 80 & 3.8 \\
\hline IL-8 & 0.752 & 0.063 & $>3.5$ & 75.7 & 76.7 & 3.2 \\
\hline Anti-TBGL IgG & 0.726 & 0.063 & $>4$ & 67.6 & 70 & 2.3 \\
\hline Anti-TBGL IgA & 0.667 & 0.069 & $>1.7$ & 70.3 & 66.7 & 2.1 \\
\hline
\end{tabular}

most discriminatory variables among groups. OPN was identified as having the highest discriminatory capacity, followed by IP-10, neutrophils, IL-6, IL- 8 , TNF- $\alpha$, MCP-1, platelets, gal-9, and WBC. Using a combination of these markers, all healthy individuals and $96.3 \%$ of TB patients were correctly classified.

\section{Discussion}

Despite its low sensitivity, AFB staining is often used in attempt to differentiate mycobacterial infection from other pulmonary bacterial infections. Bacterial culture methods, the QuantiFERON TB test, and PCR have improved the 
sensitivity and sensitivity of TB detection but are timeconsuming and require trained laboratory workers. Recently, we developed a LAMP method for detecting MTB with excellent accuracy in one hour [3]. Here, we confirmed MTB infection in all of our samples by LAMP; bacterial cultures were not performed because previous studies showed that MTB-LAMP sensitivity in culture positive samples reached $100 \%$ and that specificity in culture negative samples was 94.2\% [3]. Moreover, the research group from Thailand found that, in the clinical unknown samples test, the sensitivity of LAMP method was $98.92 \%$ and the specificity was $100 \%$ compared to those of the standard culture assay [21]. Samples were also studied by spoligotyping to determine the most prevalent circulating strains in Metro Manila. We found that all of our TB patients were infected with a Manila type of MTB and this finding of a uniformed genotype of MTB was rather surprising. The arrival of Chinese, Japanese, and Spanish groups may have influenced the acquisition of various MTB genotypes in the region; however, little information is available regarding prevalent MTB genotypes circulating in the Philippines.

The role of matricellular proteins supporting TB infection is not well studied. We found high plasma levels of two matricellular proteins, OPN, and, for the first time, gal-9 in treatment-naïve TB patients. Matricellular proteins are secreted into the extracellular matrix environment but do not play a primary structural role in this location and regulate an unusually diverse array of cellular functions, including cell adhesion, shape, migration, differentiation, proliferation, and inflammatory responses [22]. It has been proposed that matricellular proteins enter inflamed tissue and become immobilized at that site to generate signals for phagocytosis and chemotaxis of inflammatory cells [23]. OPN is highly expressed in tuberculous granuloma and supports granuloma formation via its functions as a chemoattractant cytokine [24]. OPN and gal-9 may be produced by activated circulating immune cells, but a more plausible explanation is that they are released into the circulation from tissue sites. Our unpublished data showed elevated pleural fluid/plasma ratios of gal-9 and OPN in a TB patient, and Inomata et al. showed that OPN levels increase proportionally with the extent of lung lesions [25]. We evaluate differences between patients with and without granuloma formation, factoring in the extent of lesions and other clinical parameters; however, we did not observe any statistical correlations, probably because radiological findings with most patients were not suggestive of granulomas. We found that the OPN and gal-9 correlated with IL- 8 and IP-10 and we hypothesize that OPN and gal-9 activate the expression of chemokines and cytokines like IP10 , TNF- $\alpha$, IL-6, and IL-8 in macrophages, helping to recruit immune cells to the site of MTB infection. This speculation is supported by observations from Bai et al., who found that matricellular protein CCN1 enhances the expression of TNF- $\alpha$, IL- $1 \beta$, IL-6, IFN- $\gamma$, MIP- $1 \alpha$, and IP-10 in murine macrophages through its receptors $\alpha_{\mathrm{M}} \beta_{2}$ and syndecan-4 [22]. IP-10 is produced at sites of inflammation and its blood levels reportedly correlate with the extent of inflammatory process $[26,27]$. In contrast with low circulating levels of IFN$\gamma$, the high levels of IP-10 in the blood produced by antigenstimulated cells make this chemokine a promising candidate biomarker for MTB infection, even in HIV-infected individuals $[28,29]$. We found that patients with high plasma IP10 reported loss of appetite, but such a tendency was not observed with other biomarkers. Juffermans et al. had similar observations, where IP-10 but not IL-8, MIP-1b, or MCP-1 was associated with loss of appetite and fever [30].

Neutrophils, one of the most predictive markers of active TB infection, were found to have the largest influence on WBC elevation in TB patients. Neutrophils are generally thought to have strong activity against infectious agents. Higher neutrophil counts were observed in TB patients and were associated with poorer prognosis [31]. Neutrophils are attracted by various cytokines and chemokines, including IL-8, and quickly accumulate at sites of mycobacterial infection [32-34]. We also observed IL-8 elevation in TB patients, which may be due to hyperproduction by stimulated macrophages, by epithelial cells in lungs, or even by neutrophils themselves, although no correlation with neutrophils was observed. Previous study showed that low lymphocyte count is suggestive of extension of the disease by new tubercle formation, but switch to lymphocytosis shows tendency to healing [35]. Therefore, careful evaluation of hematological changes may have diagnostic value.

It is generally accepted that cell-mediated immunity has a critical role in the protection against MTB. However, MTB is a facultative intracellular pathogen, which has both intracellular and extracellular phases in their infectious cycle. Recent studies showed that immunization using monoclonal and polyclonal antibodies and mucosal vaccination trials demonstrate convincingly the essential interdependence and synergy between cell-mediated immunity and humoral immunity [36]. WHO warned against the utility of current serological tests in the immunodiagnosis of TB and strongly recommended that they must not be used for the diagnosis of pulmonary and extrapulmonary $\mathrm{TB}$, but we believe that detection of anti-TBGL antibody titers may serve as a warrant signal of MTB infection.

In our study, TB patients represented a small group of individuals who underwent the examination due to suspected TB disease. In all of the patients, only Manila genotype was identified; therefore, it would be of interest to study the immunological profile of TB patients infected by other MTB genotypes. Future, larger sample size studies should prove the application of LAMP method and proposed biomarkers in TB research.

In conclusion, LAMP is a simple and low-cost method for the direct detection of MTB in patients' sputa. Moreover, the LAMP method together with spoligotyping method serves as sensitive, accurate tools for TB diagnosis and for monitoring prevalent lineages of TB in certain regions. Increased IP-10, $\mathrm{OPN}$, and neutrophils levels best reflect the acute stage of TB infection, and measuring their fluctuations may provide a reasonable basis for determining TB severity and prognosis.

\section{Conflict of Interests}

The authors declare that there is no conflict of interests regarding the publication of this paper. 


\section{Authors' Contribution}

Beata Shiratori and Susan Leano wrote the paper, preformed biomarker measurements and data analysis, and equally contributed to the work. Chie Nakajima evaluated LAMP and spoligotyping results; Haorile Chagan-Yasutan performed Luminex assay; Toshiro Niki measured gal-9; Yugo Ashino, Yasuhiko Suzuki, Elisabeth Telan, and Toshio Hattori contributed to the research planning and conducted the work. Toshio Hattori is the guarantor.

\section{Acknowledgments}

This work was supported by the Scientific Research Expenses for Health and Welfare program from the Ministry of Health, Labour, and Welfare, Japan (TH), the Science and Technology Research Partnership for Sustainable Development from the Japan Science and Technology Agency, Japan (YS), and collaborative funding from the Research Centre for Zoonosis Control at Hokkaido University. The authors are grateful to Kyowa Medex, Japan, for providing Determiner TBGL Antibody ELISA Kit.

\section{References}

[1] World Health Organization, "Global tuberculosis report 2013," Tech. Rep. WHO/HTM/TB/2013.11, World Health Organization, Geneva, Switzerland, 2014, http://www.who.int/tb/publications/global_report/en/.

[2] V. Schmidt, S. Schneider, J. Schlömer, M.-E. KrautwaldJunghanns, and E. Richter, "Transmission of tuberculosis between men and pet birds: a case report," Avian Pathology, vol. 37, no. 6, pp. 589-592, 2008.

[3] B. D. Pandey, A. Poudel, T. Yoda et al., "Development of an inhouse loop-mediated isothermal amplification (LAMP) assay for detection of Mycobacterium tuberculosis and evaluation in sputum samples of Nepalese patients," Journal of Medical Microbiology, vol. 57, no. 4, pp. 439-443, 2008.

[4] Y. Koguchi, K. Kawakami, K. Uezu et al., "High plasma osteopontin level and its relationship with interleukin-12-mediated type $1 \mathrm{~T}$ helper cell response in tuberculosis," American Journal of Respiratory and Critical Care Medicine, vol. 167, no. 10, pp. 1355-1359, 2003.

[5] T. Uede, "Osteopontin, intrinsic tissue regulator of intractable inflammatory diseases," Pathology International, vol. 61, no. 5, pp. 265-280, 2011.

[6] U. R. Siddiqi, H. Chagan-Yasutan, C. Nakajima et al., "Distinct clinical features in nontuberculous mycobacterial disease with or without latent tuberculosis infection," Tohoku Journal of Experimental Medicine, vol. 226, no. 4, pp. 313-319, 2012.

[7] H. Chagan-Yasutan, H. Saitoh, Y. Ashino et al., "Persistent elevation of plasma osteopontin levels in HIV patients despite highly active antiretroviral therapy," Tohoku Journal of Experimental Medicine, vol. 218, no. 4, pp. 285-292, 2009.

[8] Y. Koguchi, K. Kawakami, S. Kon et al., "Penicillium marneffei causes osteopontin-mediated production of interleukin-12 by peripheral blood mononuclear cells," Infection and Immunity, vol. 70, no. 3, pp. 1042-1048, 2002.

[9] P. Jayaraman, I. Sada-Ovalle, S. Beladi et al., "Tim3 binding to galectin-9 stimulates antimicrobial immunity;" The Journal of Experimental Medicine, vol. 207, no. 11, pp. 2343-2354, 2010.
[10] I. Sada-Ovalle, L. Chávez-Galán, L. Torre-Bouscoulet et al., "The Tim3-Galectin 9 pathway induces antibacterial activity in human macrophages infected with mycobacterium tuberculosis," The Journal of Immunology, vol. 189, no. 12, pp. 5896-5902, 2012.

[11] Y. Qiu, J. Chen, H. Liao et al., "Tim-3-expressing $\mathrm{CD} 4^{+}$and $\mathrm{CD}^{+} \mathrm{T}$ cells in human tuberculosis (TB) exhibit polarized effector memory phenotypes and stronger anti-TB effector functions," PLoS Pathogens, vol. 8, no. 11, Article ID e1002984, 2012.

[12] M. Ruhwald, M. G. Aabye, and P. Ravn, "IP-10 release assays in the diagnosis of tuberculosis infection: current status and future directions," Expert Review of Molecular Diagnostics, vol. 12, no. 2, pp. 175-187, 2012.

[13] S.-Y. Eum, J.-H. Kong, M.-S. Hong et al., "Neutrophils are the predominant infected phagocytic cells in the airways of patients with active pulmonary TB," Chest, vol. 137, no. 1, pp. 122-128, 2010.

[14] M. P. R. Berry, C. M. Graham, F. W. McNab et al., "An interferoninducible neutrophil-driven blood transcriptional signature in human tuberculosis," Nature, vol. 466, no. 7309, pp. 973-977, 2010.

[15] I. Sugawara, T. Udagawa, and H. Yamada, "Rat neutrophils prevent the development of tuberculosis," Infection and Immunity, vol. 72, no. 3, pp. 1804-1806, 2004.

[16] J. Pedrosa, B. M. Saunders, R. Appelberg, I. M. Orme, M. T. Silva, and A. M. Cooper, "Neutrophils play a protective nonphagocytic role in systemic Mycobacterium tuberculosis infection of mice," Infection and Immunity, vol. 68, no. 2, pp. 577-583, 2000.

[17] X. Zhang, L. Majlessi, E. Deriaud, C. Leclerc, and R. Lo-Man, "Coactivation of Syk kinase and MyD88 adaptor protein pathways by bacteria promotes regulatory properties of neutrophils," Immunity, vol. 31, no. 5, pp. 761-771, 2009.

[18] E. B. Eruslanov, I. V. Lyadova, T. K. Kondratieva et al., "Neutrophil responses to Mycobacterium tuberculosis infection in genetically susceptible and resistant mice," Infection and Immunity, vol. 73, no. 3, pp. 1744-1753, 2005.

[19] J. Kamerbeek, L. Schouls, A. Kolk et al., "Simultaneous detection and strain differentiation of Mycobacterium tuberculosis for diagnosis and epidemiology," Journal of Clinical Microbiology, vol. 35, no. 4, pp. 907-914, 1997.

[20] K. Brudey, J. R. Driscoll, L. Rigouts et al., "Mycobacterium tuberculosis complex genetic diversity: mining the fourth international spoligotyping database (SpolDB4) for classification, population genetics and epidemiology," BMC Microbiology, vol. 6, article 23, 2006.

[21] T. Kaewphinit, N. Arunrut, W. Kiatpathomchai, S. Santiwatanakul, P. Jaratsing, and K. Chansiri, "Detection of Mycobacterium tuberculosis by using loop-mediated isothermal amplification combined with a lateral flow dipstick in clinical samples," BioMed Research International, vol. 2013, Article ID 926230, 6 pages, 2013.

[22] T. Bai, C.-C. Chen, and L. F. Lau, "Matricellular protein $\mathrm{CCN1}$ activates a proinflammatory genetic program in murine macrophages," Journal of Immunology, vol. 184, no. 6, pp. 32233232, 2010.

[23] T. L. Adair-Kirk and R. M. Senior, "Fragments of extracellular matrix as mediators of inflammation," International Journal of Biochemistry and Cell Biology, vol. 40, no. 6-7, pp. 1101-1110, 2008. 
[24] G. J. Nau, P. Guilfoile, G. L. Chupp et al., "A chemoattractant cytokine associated with granulomas in tuberculosis and silicosis," Proceedings of the National Academy of Sciences of the United States of America, vol. 94, no. 12, pp. 6414-6419, 1997.

[25] S.-I. Inomata, N. Shijubo, S. Kon et al., "Circulating interleukin18 and osteopontin are useful to evaluate disease activity in patients with tuberculosis," Cytokine, vol. 30, no. 4, pp. 203-211, 2005.

[26] M. Liu, S. Guo, J. M. Hibbert et al., "CXCL10/IP-10 in infectious diseases pathogenesis and potential therapeutic implications," Cytokine and Growth Factor Reviews, vol. 22, no. 3, pp. 121-130, 2011.

[27] M. Baggiolini, B. Dewald, and B. Moser, "Human chemokines: an update," Annual Review of Immunology, vol. 15, pp. 675-705, 1997.

[28] M. Ruhwald, M. Bjerregaard-Andersen, P. Rabna, J. EugenOlsen, and P. Ravn, "IP-10, MCP-1, MCP-2, MCP-3, and IL-1RA hold promise as biomarkers for infection with $\mathrm{M}$. tuberculosis in a whole blood based T-cell assay," BMC Research Notes, vol. 2, article 19, 2009.

[29] D. Goletti, A. Raja, B. S. A. Kabeer et al., "Is IP-10 an accurate marker for detecting M. tuberculosis-specific response in HIVinfected persons," PLoS ONE, vol. 5, no. 9, Article ID e12577, 2010.

[30] N. P. Juffermans, A. Verbon, S. J. H. Van Deventer et al., "Elevated chemokine concentrations in sera of human immunodeficiency virus (HIV)-seropositive and HIV-seronegative patients with tuberculosis: a possible role for mycobacterial lipoarabinomannan," Infection and Immunity, vol. 67, no. 8, pp. 4295-4297, 1999.

[31] P. F. Barnes, J. M. Leedom, L. S. Chan et al., "Predictors of short-term prognosis in patients with pulmonary tuberculosis," Journal of Infectious Diseases, vol. 158, no. 2, pp. 366-371, 1988.

[32] A. M. Wengner, S. C. Pitchford, R. C. Furze, and S. M. Rankin, "The coordinated action of G-CSF and ELR + CXC chemokines in neutrophil mobilization during acute inflammation," Blood, vol. 111, no. 1, pp. 42-49, 2008.

[33] N. Mukaida, A. Harada, and K. Matsushima, "Interleukin8 (IL-8) and monocyte chemotactic and activating factor (MCAF/MCP-1), chemokines essentially involved in inflammatory and immune reactions," Cytokine and Growth Factor Reviews, vol. 9, no. 1, pp. 9-23, 1998.

[34] Y. Zhang, M. Broser, H. Cohen et al., "Enhanced interleukin8 release and gene expression in macrophages after exposure to Mycobacterium tuberculosis and its components," Journal of Clinical Investigation, vol. 95, no. 2, pp. 586-592, 1995.

[35] G. Akpek, "Hematologic findings in mycobacterial infections among immunosuppressed and immunocompetent patients," in Tuberculosis, M. M. Madkour, Ed., pp. 213-230, Springer, Berlin, Germany, 2004.

[36] F. Abebe and G. Bjune, "The protective role of antibody responses during Mycobacterium tuberculosis infection," Clinical and Experimental Immunology, vol. 157, no. 2, pp. 235-243, 2009. 


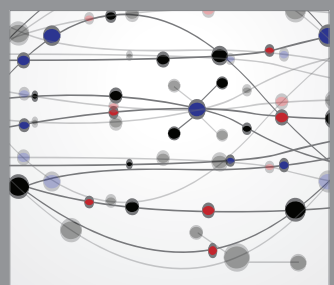

The Scientific World Journal
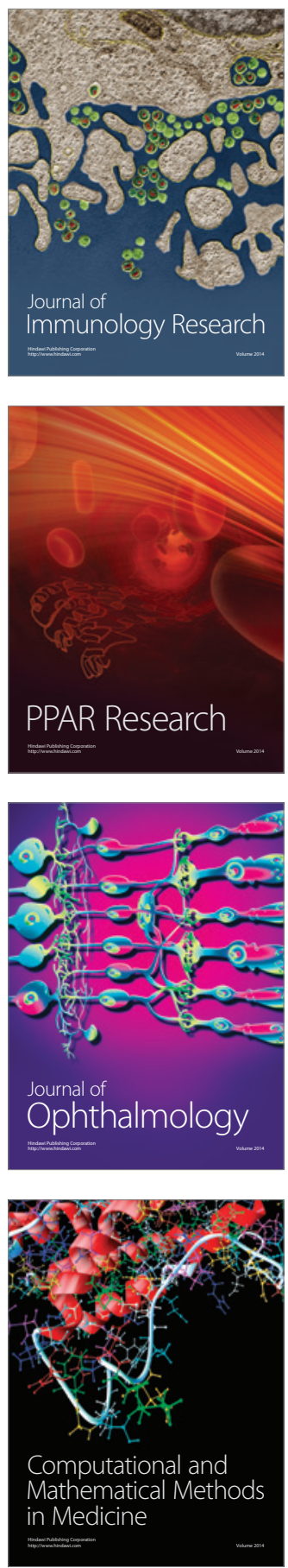

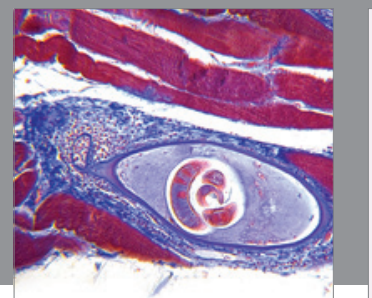

Gastroenterology

Research and Practice
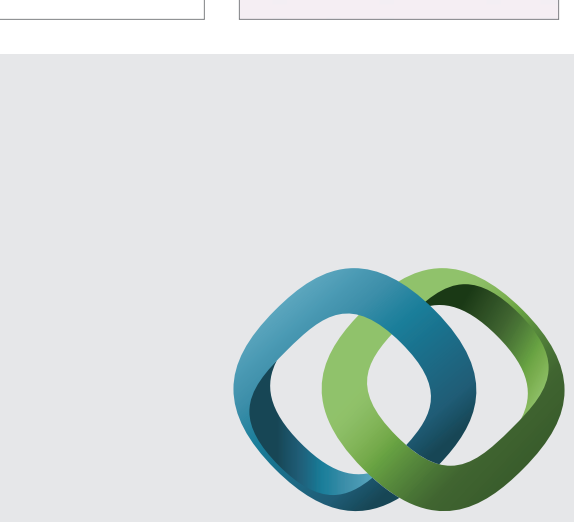

\section{Hindawi}

Submit your manuscripts at

http://www.hindawi.com
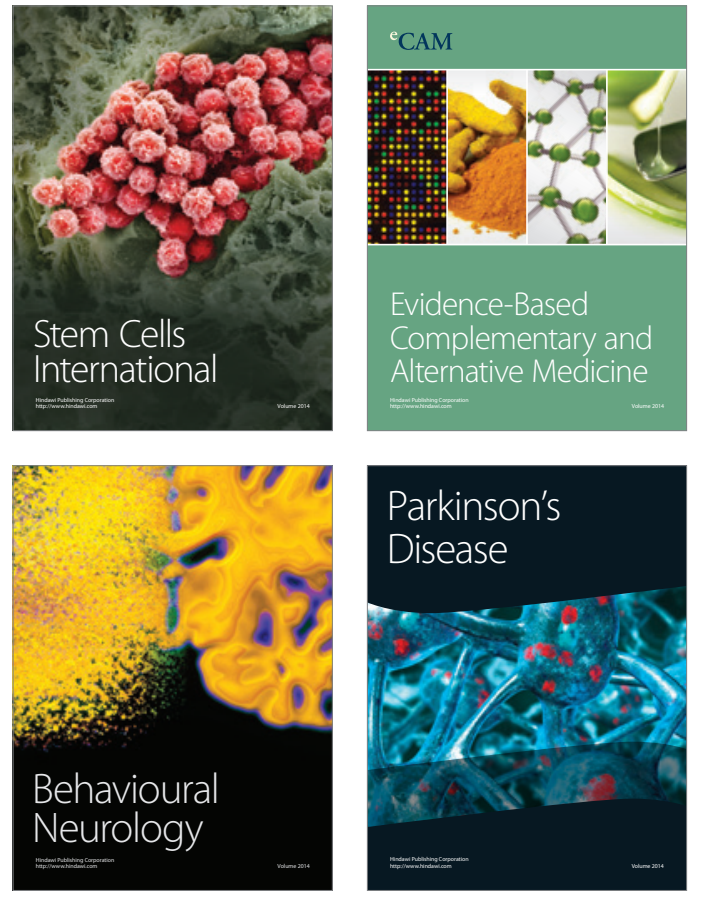
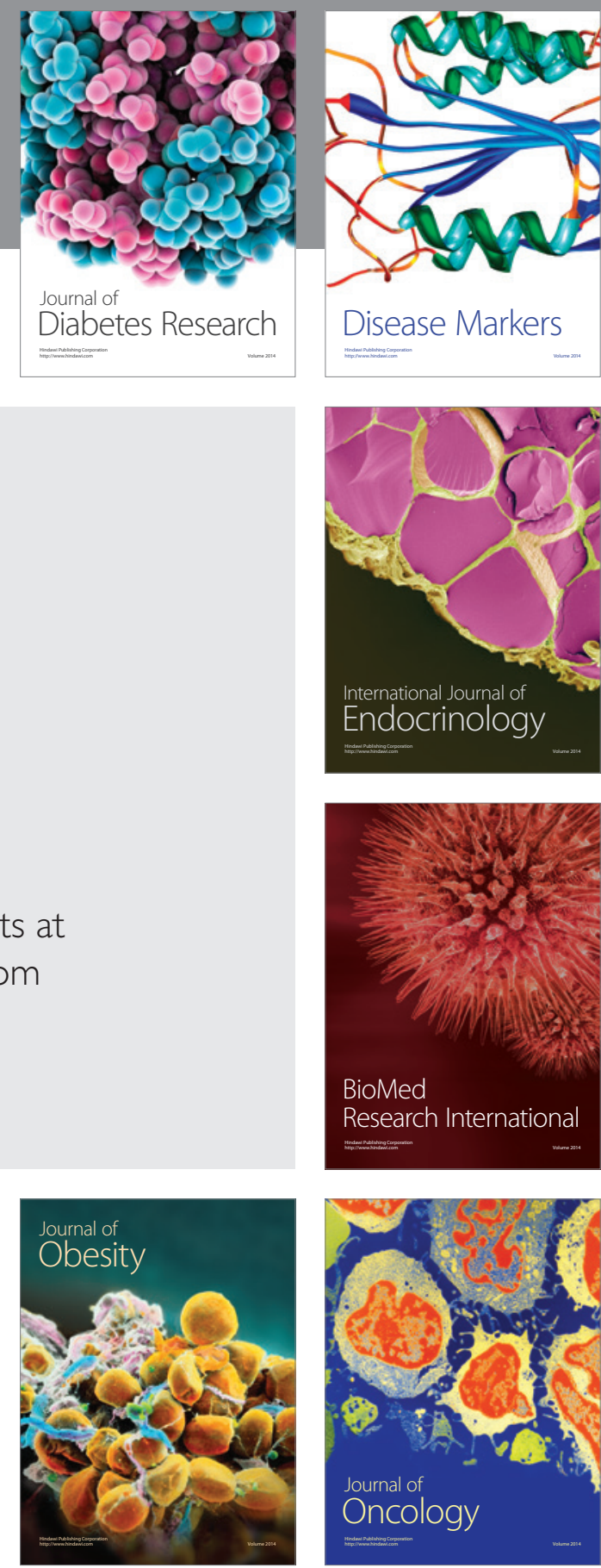

Disease Markers
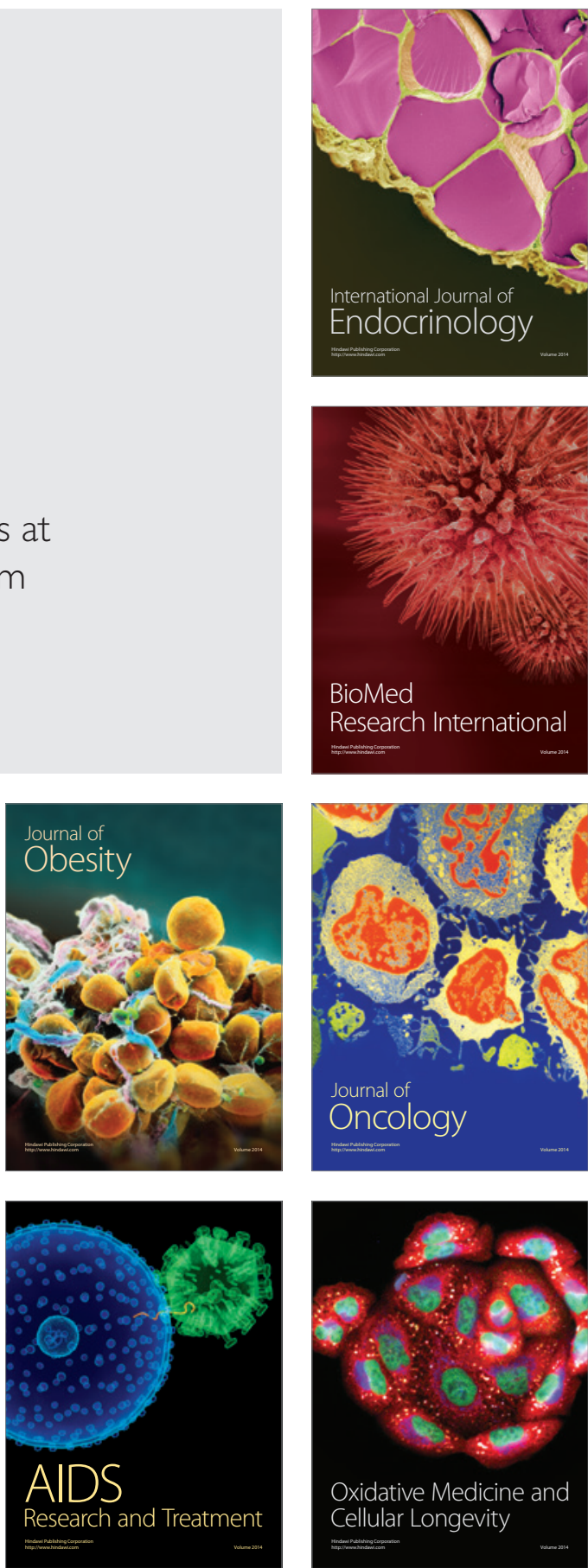